\title{
Revision rate of reconstructions in surgically treated diaphyseal metastases of bone
}

\author{
Gilber Kask ${ }^{\mathrm{a}}$, , Jyrki Nieminen ${ }^{\mathrm{b}}$, Michael C. Parry ${ }^{\mathrm{c}}$, Vincent van Iterson ${ }^{\mathrm{d}}$ e, \\ Toni-Karri Pakarinen ${ }^{\mathrm{b}}$, Maire Ratasvuori ${ }^{\mathrm{f}}$, Minna K. Laitinen ${ }^{\mathrm{d}, \mathrm{e}}$ \\ a Department of Orthopaedics and Traumatology, Tampere University Hospital, Tampere, Finland \\ ${ }^{\mathrm{b}}$ Coxa Hospital for Joint Replacement, Tampere, Finland \\ ${ }^{\mathrm{c}}$ Royal Orthopaedic Hospital, Birmingham, UK \\ ${ }^{\mathrm{d}}$ Department of Orthopaedics and Traumatology, Helsinki University Hospital, Helsinki, Finland \\ e University of Helsinki, Helsinki, Finland \\ ${ }^{\mathrm{f}}$ Department of Hand Surgery, Helsinki University Hospital, Helsinki, Finland
}

\section{A R T I C L E I N F O}

\section{Article history:}

Received 19 April 2019

Received in revised form

5 May 2019

Accepted 19 May 2019

Available online 21 May 2019

\section{Keywords:}

Neoplasms

Diaphysis

Femur

Humerus

Fractures

Spontaneous

\begin{abstract}
A B S T R A C T
Introduction: Skeletal metastases can weaken the bone, necessitating surgery, and surgical treatment options vary. The aim of this study was to investigate the revision rate of reconstructions in surgically treated diaphyseal skeletal metastases.

Materials and methods: Between 2000 and 2018 at Helsinki and Tampere university hospitals in Finland a total of 164 cases with diaphyseal skeletal metastases were identified from a prospectively maintained database. Tumor location was humerus, femur, and tibia in 106 (65\%), 53 (32\%), and 5 (3.0\%) cases, respectively. A total of $82(50 \%)$ cases were treated with intramedullary nailing (IMN), 73 (45\%) with IMN and cementation, and 9 (5\%) with another technique.

Results: In the upper extremity, implant survival (IS) was $96.4 \%$ at 1, 2, and 5 years; in the lower extremity, it was $83.8 \%, 69.1 \%$, and $57.6 \%$ at 1,2 , and 5 years, respectively. Lower extremity IS for impending lesions was $100 \%$ at 1,2 , and 5 years, and in cases operated for true pathologic fracture, it was $71.6 \%$, $42.9 \%$, and $21.5 \%$ at 1,2 , and 5 years, respectively. In IMN cases without cement, the complication rate was $16 \%(13 / 82)$ when compared to $6 \%(4 / 73)$ in IMN cases with cementation.

Discussion: We would advocate for early intervention in patients with metastatic bone disease affecting the femur rather that watchful waiting with the risk for fracture and the need for urgent intervention. However, this choice must be balanced against the underlying risk of surgical intervention in a potentially fragile population with often limited prognoses.
\end{abstract}

(ㄷ) 2019 Published by Elsevier Ltd.

\section{Introduction}

Skeletal metastases are the most common destructive bone conditions encountered by orthopedic oncologists. In patients with disseminated cancer, bone is the third most common site of metastatic disease after the lung and the liver. In the appendicular skeleton, the femur is the most frequent site for metastases [1], and

\footnotetext{
* Corresponding author. Department of Orthopaedics and Traumatology, Tampere University Hospital, Tampere, Teiskontie 35, 33521, Tampere, Finland.

E-mail addresses: gilber.kask@pshp.fi (G. Kask), jyrki.nieminen@coxa.fi (J. Nieminen), michael.parry3@nhs.net (M.C. Parry), vincent.iterson-van@hus.fi (V. van Iterson), tkpakarinen@me.com (T.-K. Pakarinen), maire.ratasvuori@hus.fi (M. Ratasvuori), minna.laitinen@hus.fi (M.K. Laitinen).
}

the most common primary cancer is breast followed by lung, prostate, renal cell carcinoma (RCC), and thyroid cancer [2]. As the incidence of cancer and survival of disseminated cancer patients has increased, so has the incidence of skeletal-related events involving metastasis [3].

Metastatic lesions can weaken the bone and may require surgery. The indications for surgery of long bone metastases are intractable pain and an impending or actual pathologic fracture [2]. Surgical indications differ among regions; $75 \%$ of surgeries for skeletal metastasis treatment in the United States are performed for impending fracture, but in Scandinavian countries, impending fracture was the indication for only $18 \%$ of the cases [4,5]. There is no consensus on the definition of impending fracture. Impending fracture refers to the state of a bone where a pathological fracture 
appears almost certain if no preventative action is taken. Several scoring systems have been developed, including Mirels' scoring system [6], Harrington's criteria [7] and scoring from van der Linden at al [8]. However, there is currently no definite and valuable tool that can be used universally to objectively quantify the risk [9]. Because skeletal metastases almost always indicate that the disease is incurable, surgery for skeletal metastases is palliative, with the main purpose of stabilizing the bone and preserving the patient's independence through immediate functional restoration in a single procedure, without need for revision surgery [2,10-12].

Most procedures performed for skeletal metastases are in the metaphyseal area. The management of metaphyseal metastases varies, and most surgeons treat these patients according to their own preferential standard practice for pathologic fractures [2]. Surgical alternatives to metaphyseal metastases range from stabilization with an intramedullary nailing (IMN) to resection and endoprosthetic reconstruction (EPR), depending on estimated survival, because the device chosen should last the remainder of the patient's life [13].

Diaphyseal metastases in the bone are less common, representing only approximately $20 \%$ of surgically treated skeletal metastases [1]. Surgical alternatives are limited, and nailing offers a simple, relatively quick and inexpensive solution, permitting immediate delivery of radiotherapy without the risk of wound compromise. With time, however, intramedullary nails carry a risk of failure. Because of tumor growth or radiotherapy, pathologic fractures do not heal, healing takes too much time, or healing takes place with an insufficient bone callus. Cement augmentation is used for additional mechanical stability of the fixation and is reported to decrease mechanical failure of the implants [13-15].

Because of the rarity of pathologic fractures of diaphyseal metastases, however, information is lacking in this field. To our knowledge, no groups have reported the outcome and revision rate of surgically treated diaphyseal skeletal metastases. Therefore, the aims of this study were to (i) investigate the implant survival (IS) of reconstructions, (ii) present complication rates, and (iii) investigate patient survival in surgically treated diaphyseal skeletal metastases.

\section{Material and methods}

\section{Study design}

This multi-center retrospective study included 164 fractures or impending fractures. Following institutional ethical review board approval, patients who were diagnosed and surgically treated for a metastatic long-bone diaphyseal lesion in the diaphyseal area of thick cortical bone between January 2000 and January 2018 at the Helsinki University Hospital, Helsinki, Finland, and Tampere University Hospital, Tampere, Finland, were identified from prospectively maintained databases. All the other anatomical locations, including metaphyseal area, were excluded. Details of the collected data included the primary tumor, site, type of operation (IMN, plate-screw fixation, EPR, or a combination), fracture type (impending or true pathologic fractures), metastatic load, sex, age, complications, and oncological and surgical outcomes, including patient survival and IS. IS was defined as revisions for any reason as the final result. Impending fracture was considered when patient had a painful medullary lytic lesion resulting a significant reabsorption of the cortical thickness, defined by the experienced surgeon.

\section{Demographics}

A total of 164 procedures were performed in 146 patients. The study comprised 67 (41\%) men and 97 (59\%) women with a mean age of 70 years (range, 38-94) at the time of surgery. The primary tumor was breast cancer in 50 (30.5\%), myeloma in 32 (19.5\%), lung cancer in 21 (12.8\%), RCC in 19 (11.6\%), prostate cancer in $16(9.8 \%)$, and other in $26(15.8 \%)$ cases. Of the 164 lesions, 106 (65\%) were in the humerus, $53(32 \%)$ in the femur, and $5(3 \%)$ in the tibia. Nine (6\%) cases were solitary synchronous skeletal metastases. In 31 cases (19\%), reconstruction was undertaken for impending fracture indicated by significant bone destruction, intractable pain, and loss of function, and in 129 (79\%) cases for true pathologic fracture. In 4 (2\%) cases, data were missing.

The mean lag time from diagnosis of the primary malignancy to diagnosis of skeletal metastases was 46 months (range, 0-396). The mean lag time from diagnosis of skeletal metastases to surgery of metastases was 18 months (range, 0-241). Mean follow-up was 12.4 months (range, $0-184)$. A total of $83 \%(121 / 145)$ of the patients died during follow-up, and 47\% (68/145) died within 6 months after surgery. Patient characteristics are summarized in Table 1.

\section{Surgical procedures}

A total of 61 cases (37\%) were operated in the Helsinki University Hospital and 103 cases (63\%) in the Tampere University Hospital, in Finland. Procedures were performed by orthopedic oncologists (orthopedic surgeon been specialized in the treatment of bone tumors) or experienced orthopedic surgeons (minimum of five years' experience).

Depending on the site of the metastatic lesion, different surgical procedures were used. Diaphyseal lesions were treated by long static IMN with or without cementation, plate-screw fixation, or EPR. The implant used and the use of cement was based on surgeon preference. In femur, only long intramedullary nails were used. In all cases of IMN fixation without cementation, nails were locked in static mode. Postoperative care and rehabilitation varied among patients, depending on their health status, but with the goal of weight-bearing as tolerated.

In the humerus, the operative method was IMN without cement in 41 cases (39\%), IMN with cement in 59 cases (56\%), osteosynthesis with plate fixation in 4 cases (4\%), and EPR in 2 cases (2\%). In femur, IMN without cement was used in 39 patients (74\%), IMN with cement in 11 patients (21\%), and EPR in 3 cases (6\%). In tibia, 2 cases were treated with IMN without cement and 3 cases with IMN with cement. In femur and humerus, the reason for surgery was an impending fracture in 21 of 51 (41\%) and in 8 of 104 (8\%), respectively. In 4 cases, the data were missing.

\section{Statistical analysis}

Descriptive statistics were used to present the demographic data, and the Kaplan-Meier method was applied for patient and IS. Survival rates were calculated from the date of surgery to the most recent follow-up, death, or IS to revision surgery. Univariate analysis was performed by comparing groups with the log-rank test with subsequent multivariate Cox proportional hazard analysis of significant variables to identify independent predictors of patient survival or IS. The Chi-square test or Fisher's exact test was used to compare variables between groups and the Kruskal-Wallis test for means between groups. A p-value $<0.05$ was considered significant. Statistical analysis was done using SPSS Statistics 23.0 (IBM, New York, USA).

\section{Results}

Overall IS was $91.5 \%$ (95\% confidence interval (CI), 85\%-98\%) at 1 year, $81.9 \%$ (95\% CI, 68\%-96\%) at 2 years, and 74.4\% (95\% CI, 56\%$93 \%$ ) at 5 years (Fig. 1). When stratified by location, in the upper 
Table 1

Characteristics of cases (values are presented in number of cases).

\begin{tabular}{|c|c|c|c|c|}
\hline Characteristics & Total & Nailing & Nailing with cement & Other \\
\hline Eligible cases & 164 & $82(50.0 \%)$ & $73(44.5 \%)$ & $9(5.4 \%)$ \\
\hline \multicolumn{5}{|l|}{ Sex } \\
\hline Female & $97(59.1 \%)$ & & & \\
\hline Male & $67(40.9 \%)$ & & & \\
\hline \multicolumn{5}{|l|}{ Location } \\
\hline Humerus & $106(64.6 \%)$ & $41(38.7 \%)$ & $59(55.7 \%)$ & $6(5.7 \%)$ \\
\hline Femur & $53(32.3 \%)$ & $39(73.6 \%)$ & $11(20.8 \%)$ & $3(5.7 \%)$ \\
\hline Tibia & $5(3.0 \%)$ & $2(40.0 \%)$ & $3(60.0 \%)$ & - \\
\hline Mean follow-up months (range) & $12.4(0-184)$ & $15.4(0-184)$ & $9.8(0-74)$ & $7.2(0-25)$ \\
\hline Age at surgery years (range) & $70(38-94)$ & $69(38-88)$ & $70(41-94)$ & $72(42-85)$ \\
\hline \multicolumn{5}{|l|}{ Pathologic fracture } \\
\hline Present & 129 (78.7\%) & $54(41.1 \%)$ & $69(53.5 \%)$ & $7(5.4 \%)$ \\
\hline Urgent & $31(18.9 \%)$ & $25(80.6 \%)$ & $4(12.9 \%)$ & $2(6.5 \%)$ \\
\hline Data missing & $4(2.4 \%)$ & & & \\
\hline \multicolumn{5}{|l|}{ Bone metastasis } \\
\hline Solitary & $9(5.5 \%)$ & $5(55.6 \%)$ & $4(44.4 \%)$ & $0(0 \%)$ \\
\hline Multiple & $151(92.1 \%)$ & $73(48.3 \%)$ & $69(45.7 \%)$ & $9(5.9 \%)$ \\
\hline Data missing & $4(2.4 \%)$ & & & \\
\hline \multicolumn{5}{|l|}{ Pulmonary metastasis } \\
\hline Yes & $42(25.6 \%)$ & $17(40.5 \%)$ & $23(54.8 \%)$ & $2(4.8 \%)$ \\
\hline No & $122(74.6 \%)$ & $65(53.3 \%)$ & $50(41.0 \%)$ & $7(5.8 \%)$ \\
\hline \multicolumn{5}{|l|}{ Liver metastasis } \\
\hline Yes & $21(12.8 \%)$ & $7(33.3 \%)$ & $12(57.1 \%)$ & $2(9.6 \%)$ \\
\hline No & $143(87.2 \%)$ & $75(52.4 \%)$ & $61(42.7 \%)$ & $7(4.9 \%)$ \\
\hline \multicolumn{5}{|l|}{ Preoperative radiotherapy } \\
\hline Yes & $20(12.2 \%)$ & $5(25.0 \%)$ & $14(70.0 \%)$ & $1(5.0 \%)$ \\
\hline No & $144(87.8 \%)$ & $77(53.5 \%)$ & $59(41.0 \%)$ & $8(5.6 \%)$ \\
\hline \multicolumn{5}{|l|}{ Postoperative radiotherapy } \\
\hline Yes & $59(36.0 \%)$ & $31(52.5 \%)$ & $26(44.1 \%)$ & $2(3.4 \%)$ \\
\hline No & $105(64.0 \%)$ & $51(48.6 \%)$ & $47(44.8 \%)$ & $7(6.7 \%)$ \\
\hline \multicolumn{5}{|l|}{ Primary tumor } \\
\hline Breast & $50(30.5 \%)$ & $26(52.0 \%)$ & $21(42.0 \%)$ & $3(6.0 \%)$ \\
\hline Myeloma & $32(19.5 \%)$ & $16(50.0 \%)$ & $16(50.0 \%)$ & - \\
\hline Lung & $21(12.8 \%)$ & $12(57.1 \%)$ & $8(38.1 \%)$ & $1(4.8 \%)$ \\
\hline Renal cell & $19(11.6 \%)$ & $8(42.1 \%)$ & $9(47.4 \%)$ & $2(10.5 \%)$ \\
\hline Prostate & $16(9.8 \%)$ & $8(50.0 \%)$ & $8(50.0 \%)$ & - \\
\hline Other & $26(15.8 \%)$ & $12(46.2 \%)$ & $11(42.3 .0 \%)$ & $3(11.5 \%)$ \\
\hline \multicolumn{5}{|l|}{ Dead at follow-up } \\
\hline Yes & $138(84.1 \%)$ & $65(47.1 \%)$ & $67(48.6 \%)$ & $6(4.4 \%)$ \\
\hline No & $26(15.9 \%)$ & $17(65.4 \%)$ & $6(23.1 \%)$ & $3(11.5 \%)$ \\
\hline \multicolumn{5}{|l|}{ Pulmonary embolism } \\
\hline Yes & $6(3.7 \%)$ & $5(6.1 \%)$ & $0(0 \%)$ & $1(11.1 \%)$ \\
\hline \multicolumn{5}{|l|}{30 days mortality rate } \\
\hline & $25(15.2 \%)$ & $13(15.9 \%)$ & $10(13.7 \%)$ & $2(22.2 \%)$ \\
\hline
\end{tabular}

extremity, the IS was $96.4 \%$ at 1,2 , and 5 years, and in the lower extremity, the IS was $83.8 \%$ at 1 year, $69.1 \%$ at 2 years, and $57.6 \%$ at 5 years ( $\mathrm{p}=0.008$ ) (Fig. 2). Radiotherapy given either pre- or postoperatively was not a significant factor in IS $(\mathrm{p}=0.860)$.

There was no statistically significant difference between IMN with or without cement when all locations were combined. The IS for IMN without cement as a surgical method was $87.7 \%$ at 1 year, $81.8 \%$ at 2 years, and $72.2 \%$ at 5 years. When the surgical method was IMN with cement, the IS was $95.0 \%$ at 1 year and $76.0 \%$ at 2 and 5 years $(\mathrm{p}=0.329)$.

\section{Upper extremity}

In upper extremity, the IS was better in the group treated with IMN and cement when compared to the group treated with IMN without cement. IS for IMN without cement was $91.1 \%$ at 1, 2, and 5 years, and for IMN with cement, it was $100 \%$ at 1, 2, and 5 years, respectively ( $\mathrm{p}=0.112$ ) (Fig. 3 ).

\section{Lower extremity}

In lower extremity, the IS was better in the group treated for impending fracture compared to the true pathologic fractures. Lower extremity IS for impending lesions was $100 \%$ at 1,2 , and 5 years, whereas the IS for cases operated for true pathologic fracture was $71.6 \%, 42.9 \%$, and $21.5 \%$ at 1,2 , and 5 years, respectively $(p=0.017$; Fig. 4). The use of cement was not a significant factor in lower extremity IMN for increasing IS $(\mathrm{p}=0.895)$.

\section{Complications and revision}

The overall complication rate was $12 \%(18 / 155)$ in all IMN cases. In IMN cases without cement, the complication rate was $16 \%$ (13/ $82)$, compared to $6 \%(4 / 73)$ when cement was used, and the difference was statistically significant $(\mathrm{p}=0.03)$. The complication rate was $9 \%(9 / 100)$ in all upper extremity IMN cases. In the noncemented group, the complication rate was 17\% (7/41) in comparison to $3 \%(2 / 59)$ in the cemented group $(\mathrm{p}=0.014)$. Complications are summarized in Table 2 .

All upper extremity revisions were performed in patients treated with IMN without cement. All lower extremity revisions were performed in patients who were primarily operated for true pathologic fracture. 


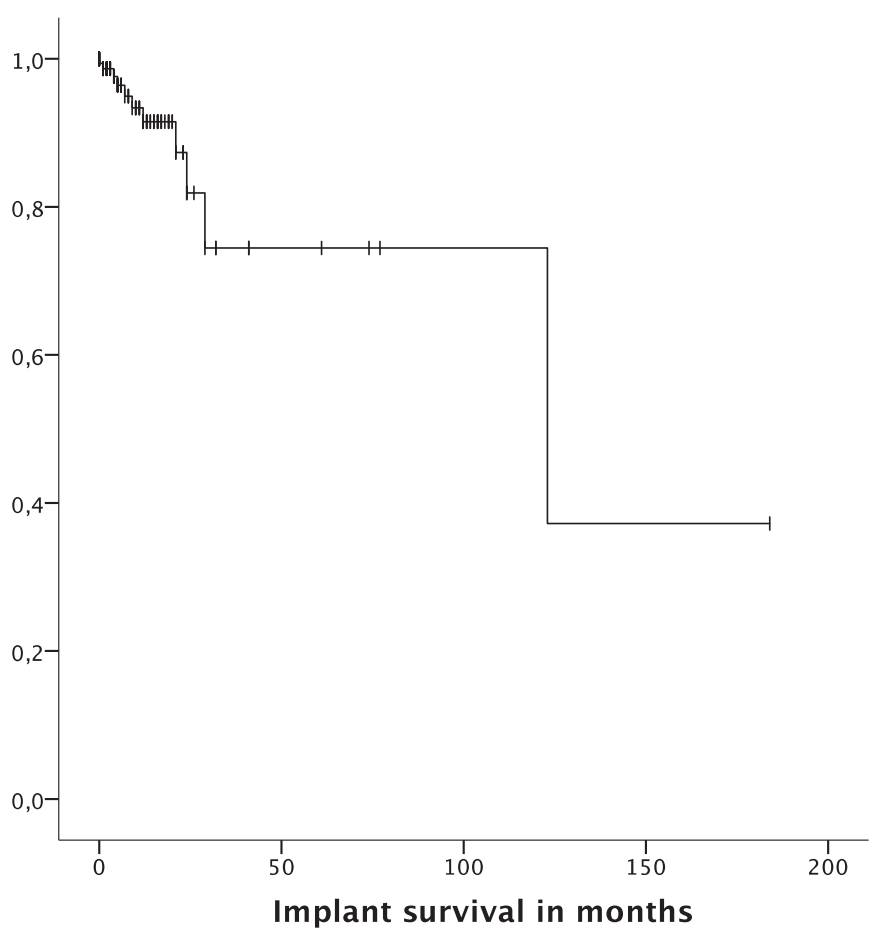

Fig. 1. Overall implant survival.

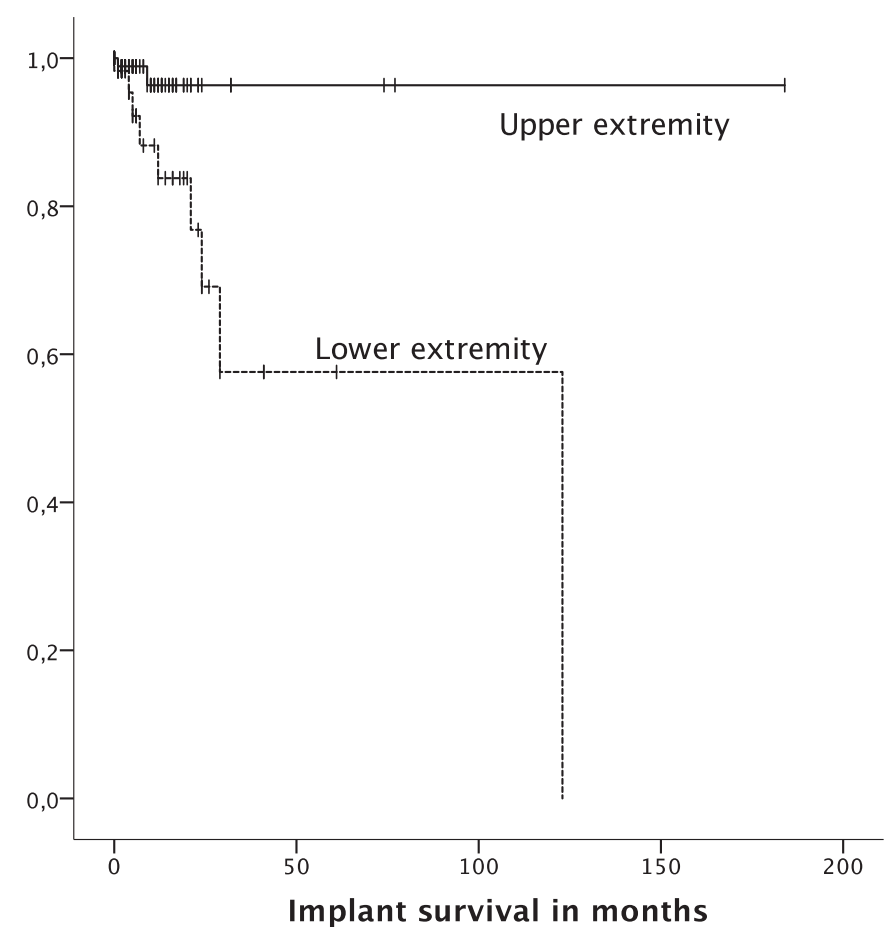

Fig. 2. Upper and lower extremities' implant survival.

Patient survival

Overall patient survival was $35.5 \%$ at 1 year, $11.1 \%$ at 2 years, and $2.7 \%$ at 5 years. In univariate analysis, significant factors affecting overall patient survival were pulmonary metastases $(\mathrm{p}=0.042)$, sex $(p=0.004)$, primary cancer $(p=0.003)$, complication $(p=0.007)$, and revision surgery $(p=0.002)$. Female sex and breast

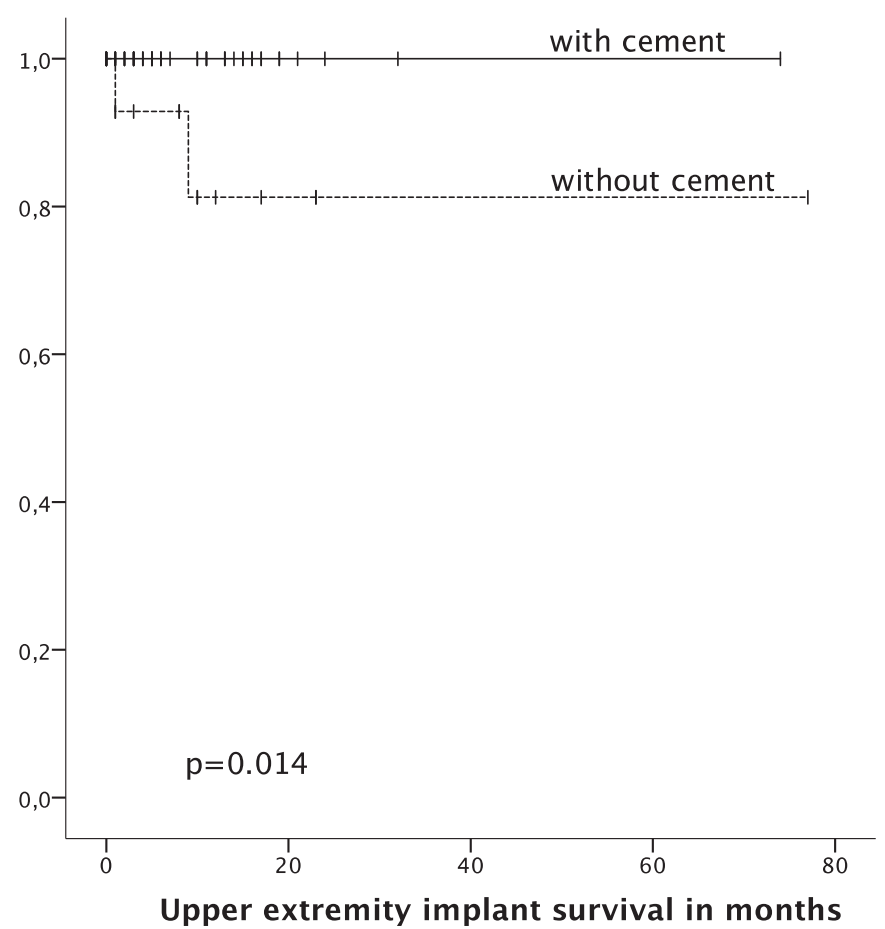

Fig. 3. Upper extremity implant survival. Intramedullary nailing with or without cement.

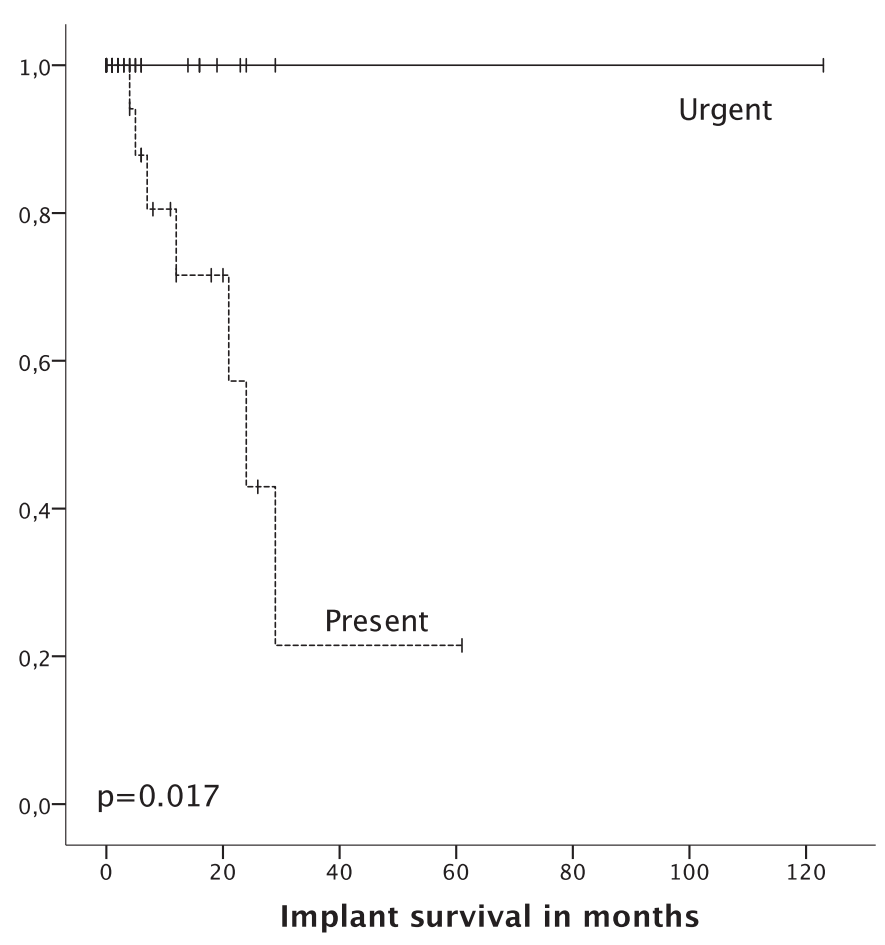

Fig. 4. Implant survival in patients with urgent or present pathologic fracture.

cancer positively affected patient survival. Pathologic fracture, when compared to impending fracture, was not a significant factor in overall survival $(\mathrm{p}=0.262)$.

Revision surgery remained a positive significant factor for overall patient survival (hazard ratio, $0.312 ; 95 \% \mathrm{CI}, 0.132-0.740$; $\mathrm{p}=0.008$ ), in multivariate analysis. The median survival time after the operation was 6 months (range, 0-184 months; mean, 21 
Table 2

Complications of the cases.

\begin{tabular}{|c|c|c|c|c|}
\hline Characteristics & Osteosynthesis problems & Tumor progression & Infection & Fracture in other part of the same bone \\
\hline \multicolumn{5}{|l|}{ Location } \\
\hline Humerus & 5 & 2 & 1 & 1 \\
\hline Femur & 5 & 3 & - & 1 \\
\hline Tibia & - & 1 & - & - \\
\hline \multicolumn{5}{|l|}{ Operation } \\
\hline I & 8 & 4 & 1 & - \\
\hline IC & 2 & 2 & - & - \\
\hline \multicolumn{5}{|c|}{ Pathologic fracture } \\
\hline Present & 10 & 5 & - & 1 \\
\hline Urgent & 1 & - & 1 & - \\
\hline
\end{tabular}

$\mathrm{I}=$ Intramedullary nailing without cementation.

IC = Intramedullary nailing with cementation.

months); it was 7 (range, 0-128; mean, 17.1) months in RCC, 15 (range, 0.1-74; mean, 16) months in myeloma, 11 (range, 0.2-185; mean, 17) months in breast cancer, 2 (range, $0-14$; mean, 3 ) months in lung cancer, and 5 (range, $0.4-51$; mean, 11 ) months in prostate cancer.

\section{Discussion}

As the biological healing of pathologic diaphyseal fractures is not to be relied on, the goal of treatment in skeletal metastases with pathologic fractures is to stabilize the lesions, regain patient mobility, and maintain the highest possible quality of life with a low revision risk. Our retrospective, multi-center study aimed to assess the prognostic factors influencing IS, complication rate, and patient survival for the treatment of diaphyseal skeletal metastases.

In non-weight-bearing extremities like the humerus, the method of surgical choice, according to the literature, does not seem to play an important role [12]; IMN [14,15] and open reduction with plate fixation [16] yield similar results and are both popular among surgeons. Plating has potentially more problems because implant choice, wound problems, and tumor progression might, in inexperienced hands, lead to failure, whereas nailing is more secure. The literature suggests advantages and disadvantages for both methods [2,17-21], but the more interesting question is the role of cement augmentation.

In our current study, in humerus, IS for IMN without cement was $91.1 \%$ at 1,2 , and 5 years, and IMN with cement was $100 \%$ at 1,2 , and 5 years, respectively. Our group has previously shown that nailing with cement augmentation reduces postoperative pain, as has been also shown by Kim et al. In their study, they could confirm that cement augmentation not only gives better pain relief but also decreases disease progression [14,22]. Even though, the difference in IS in humerus between IMN with or without cement did not reach statistical significance, we did observe a statistically significant difference in the rate of complications between these groups. The difference in the rate of revisions was most likely because the indication for revision surgery in upper extremities among these fragile patients is high. Therefore, based on our current study, in agreement with the literature, we recommend using cement augmentations in IMN when treating humeral diaphyseal pathologic fractures [14,22].

The question of when to stabilize long bone metastases, particularly those of the femur, remains a challenge. The results presented here differ from those reported previously demonstrating an advantage of IMN with cement augmentation compared to unaugmented IMN [22]. This finding may be confounded by the high number of patients with metastatic lung carcinoma included in Kim et al.'s study, a tumor type with a known poor prognosis in the presence of metastatic disease, and the proportion of impending fractures was not reported [23].

We have demonstrated a significant improvement in IS when IMN is applied to impending fracture at 1,2 , and 5 years, as compared to IMN for established pathological fracture. The IS in the complete fracture group was not improved by augmentation of fixation with cement. Actual pathologic fracture appears to make the bone very unstable and difficult to stabilize with IMN with or without cement. The poor-quality bone from disease infiltration will also contribute to the difficulty in fracture reduction and stabilization and subsequently lead to implant failure.

Conventional wisdom would suggest careful observation for impending pathological fracture, applying universally accepted though unvalidated scoring systems such as the Mirel's score, to quantify the risk of subsequent fracture [24]. Our findings suggest that early intervention for metastatic lesions of the femur results in a reduction in subsequent fracture and implant failure with a concurrent effect on quality of life for patients, many of whom are undergoing palliative treatment for advanced metastatic disease.

IMN is not without risk, and of course, an earlier intervention for such lesions would expose a number of patients to the risks of surgery, in whom no intervention might ever be required either because of mortality from advanced disease or successful systemic treatment of the underlying malignancy. Intramedullary stabilization is not without risk, either, particularly when applied to patients with underlying malignancy and exposed to its potential toxic effects [25-28]. Rather than advocate IMN of any pathological femoral lesion, we would, however, advocate a more careful assessment of patients on a case-by-case basis, taking into consideration the chemo- and radiosensitivity of the primary tumor, the projected life expectancy of the patient, and the informed wishes of the patient and their family.

One potential criticism of our findings is that the two groups differed in terms of their underlying diagnoses and demographics, the use of radiotherapy, and the definitions of impending fracture. It should be noted, however, that there were no differences in the underlying diagnoses and demographics of the two groups, and diaphyseal metastases were hardly ever solitary. Also, all cases were assessed for the risk of fracture by the two senior authors, and bias in this respect thus could be excluded.

One of the main goals in treating diaphyseal pathological lesions is to minimize the risk of complications and further revision surgery. The present study shows that the overall complication rate was $12 \%$, which is in concordance with current literature, where the rate of complications has been reported to vary from 2 to $22 \%$ $[2,20,21,29]$. Our study also showed that complication or revision surgery is a significant factor in increasing overall survival. We believe that complication or revision surgery does not improve survival but is merely a sign of patient selection because patients with better overall survival mobilize better and with the time 
develop a complication requiring revision surgery. Some skeletal metastasis, including renal cell carcinoma, are hypervascular and carry a risk of massive blood loss. Postoperative embolization is often used to reduce intraoperative blood loss [30].

Once a diagnosis of skeletal metastases is made, the disease is considered incurable, although some patients might live several years. In our study, the overall patient survival rates were $35.5 \%$, $11.1 \%$, and $2.7 \%$ at 1,2 , and 5 years, respectively. We also demonstrated that half of the patients lived less than 6 months after surgery. Our results are in accordance with the current literature of overall survival after surgery for pathologic fracture [25,31,32]. Disease-specific significant factors for decreasing survival, as indicating developed disease, were pulmonary metastases, sex, and primary cancer. Female sex and breast cancer increasingly affected patient survival. The significant difference in sex and the primary tumor is related to female sex and breast cancer, providing the great majority of cases that warranted orthopedic intervention. Breast cancer patients have improved survival as in the populationbased study, $52 \%$ of breast cancer patients were alive at 1 year after diagnosis of skeletal metastases and $26 \%$ after 3 years [31].

Our study had limitations. First, it is a retrospective study lacking any randomization. Second, we did not have any patient-related outcome measures for analyzing patient-related quality of life or functional outcome. Nevertheless, to our knowledge, this is the largest dataset that has been published. The strengths of the present study were a relatively large patient cohort and the possibility of collecting full data, thanks to our united health care system, where all patients and complications are treated in the same unit throughout the disease.

In conclusion, patient selection is always an important influencing factor and is more compelling particularly in patients with metastatic disease with limited physiological reserve. The assessment for a suitable surgical method becomes important because patient survival must be compared to a possible prophylactic procedure and the method of surgery. In locations like the proximal femur, where surgical options are more diverse, the estimated survival has more impact on implant selection. In diaphyseal metastases, however, surgical options are scarce, and although this study did not specifically address the results of revision surgery, all of the intercalary prosthesis cases were unsuccessful when used as a revision solution to treat a failed IMN; therefore, good alternatives to nailing are lacking. In the upper extremity, a well-tolerated method with a low complication risk is IMN with cement augmentation. In the lower extremity, prophylactic IMN appears to have a low complication and revision risk. The indication for prophylactic IMN is, however, undefined because burdensome overtreatment with these fragile patients should be avoided. Patients with short survival and actual pathologic fracture can be treated with IMN with or without cement augmentations, but the best surgical method for patients with actual lower extremity pathological fracture and long survival remains unsolved and needs further study.

\section{Conclusion}

We advocate early intervention for patients with metastatic bone disease affecting the femur rather than watchful waiting with the risk for fracture and the need for urgent intervention. However, this choice must be balanced by the underlying risk of surgical intervention in a potentially fragile population with often limited prognoses.

\section{Declarations of interest}

None.

\section{Ethical review committee statement}

Institutional ethical board approval.

\section{Funding}

We are grateful for financial support of this study by the Competitive Research Funding of Tampere University Hospital.

\section{References}

[1] Wedin R, Bauer HC, Wersall P. Failures after operation for skeletal metastatic lesions of long bones. Clin Orthop Relat Res 1999;(358):128-39.

[2] Errani C, et al. Treatment for long bone metastases based on a systematic literature review. Eur J Orthop Surg Traumatol 2017;27(2):205-11.

[3] Coleman RE. Clinical features of metastatic bone disease and risk of skeletal morbidity. Clin Cancer Res 2006;12(20 Pt 2):6243s-9s.

[4] Ratasvuori MWR, Keller J, Nottrott M, Zaikova O, Bergh P, Kalen A, et al. Insight opinion to surgically treated metastatic bone disease: Scandinavian Sarcoma Group Skeletal Metastasis Registry report of 1195 operated skeletal metastasis. Surg Oncol 2013;22(2):132-8.

[5] Moon B, et al. Intramedullary nailing of femoral diaphyseal metastases: is it necessary to protect the femoral neck? Clin Orthop Relat Res 2015;473(4): 1499-502.

[6] Mirels H. Metastatic disease in long bones: a proposed scoring system for diagnosing impending pathologic fractures. Clin Orthop Relat Res 1989;2003(415 Suppl):S4-13.

[7] Harrington KD. Impending pathologic fractures from metastatic malignancy: evaluation and management. Instr Course Lect 1986;35:357-81.

[8] van der Linden YM, et al. Simple radiographic parameter predicts fracturing in metastatic femoral bone lesions: results from a randomised trial. Radiother Oncol 2003;69(1):21-31.

[9] Piccioli A, Spinelli MS, Maccauro G. Impending fracture: a difficult diagnosis. Injury 2014;45(Suppl 6):S138-41.

[10] Bickels J, Dadia S, Lidar Z. Surgical management of metastatic bone disease. J Bone Joint Surg Am 2009:91(6):1503-16.

[11] Janssen SJ, et al. Outcome of operative treatment of metastatic fractures of the humerus: a systematic review of twenty three clinical studies. Int Orthop 2015;39(4):735-46.

[12] Ruggieri P, et al. Protocol of surgical treatment of long bone pathological fractures. Injury 2010;41(11):1161-7.

[13] Weber KL, et al. Management of lower-extremity bone metastasis. J Bone Joint Surg Am 2006:88(Suppl 4):11-9.

[14] Laitinen M, Nieminen J, Pakarinen TK. Treatment of pathological humerus shaft fractures with intramedullary nails with or without cement fixation. Arch Orthop Trauma Surg 2011;131(4):503-8.

[15] Dijkstra S, et al. Treatment of pathological fractures of the humeral shaft due to bone metastases: a comparison of intramedullary locking nail and plate osteosynthesis with adjunctive bone cement. Eur J Surg Oncol 1996;22(6): 621-6.

[16] Weiss KR, et al. Fixation of pathological humeral fractures by the cemented plate technique. J Bone Joint Surg Br 2011;93(8):1093-7.

[17] Hunt KJ, Gollogly S, Randall RL. Surgical fixation of pathologic fractures: an evaluation of evolving treatment methods. Bull Hosp Jt Dis 2006;63(3-4): 77-82.

[18] van Doorn R, Stapert JW. Treatment of impending and actual pathological femoral fractures with the long Gamma nail in The Netherlands. Eur J Surg 2000;166(3):247-54.

[19] Edwards SA, Pandit HG, Clarke HJ. The treatment of impending and existing pathological femoral fractures using the long gamma nail. Injury 2001;32(4): 299-306.

[20] Harvey N, et al. Endoprostheses last longer than intramedullary devices in proximal femur metastases. Clin Orthop Relat Res 2012;470(3):684-91.

[21] Wedin R, et al. Complications and survival after surgical treatment of 214 metastatic lesions of the humerus. J Shoulder Elb Surg 2012;21(8):1049-55.

[22] Kim YI, et al. Closed intramedullary nailing with percutaneous cement augmentation for long bone metastases. Bone Joint Lett J 2016;98-B(5): $703-9$.

[23] Ratasvuori M, et al. Prognostic role of en-bloc resection and late onset of bone metastasis in patients with bone-seeking carcinomas of the kidney, breast, lung, and prostate: SSG study on 672 operated skeletal metastases. J Surg Oncol 2014;110(4):360-5.

[24] Mirels H. Metastatic disease in long bones. A proposed scoring system for diagnosing impending pathologic fractures. Clin Orthop Relat Res 1989;(249): 256-64.

[25] Ratasvuori M, et al. Insight opinion to surgically treated metastatic bone disease: Scandinavian Sarcoma Group Skeletal Metastasis Registry report of 1195 operated skeletal metastasis. Surg Oncol 2013:22(2):132-8.

[26] Arvinius C, et al. Benefits of early intramedullary nailing in femoral metastases. Int Orthop 2014;38(1):129-32.

[27] Wedin R, Bauer HC. Surgical treatment of skeletal metastatic lesions of the 
proximal femur: endoprosthesis or reconstruction nail? J Bone Joint Surg $\mathrm{Br}$ 2005;87(12):1653-7.

[28] Ratasvuori M, Lassila R, Laitinen M. Venous thromboembolism after surgical treatment of non-spinal skeletal metastases - an underdiagnosed complication. Thromb Res 2016;141:124-8.

[29] Mavrogenis AF, et al. Survival analysis of patients with femoral metastases. J Surg Oncol 2012;105(2):135-41.
[30] Rossi G, et al. Selective arterial embolisation for bone tumours: experience of 454 cases. Radiol Med 2011;116(5):793-808.

[31] Weiss RJ, et al. Skeletal metastases in 301 breast cancer patients: patient survival and complications after surgery. Breast 2014;23(3):286-90.

[32] Hansen BH, et al. The scandinavian sarcoma group skeletal metastasis register Survival after surgery for bone metastases in the pelvis and extremities. Acta Orthop Scand Suppl 2004;75(311):11-5. 